\title{
LA EPIDEMIA DE CÓLERA EN GARGANTA DE BÉJAR (1885)
}

\author{
Francisco Feo Parrondo \\ Departamento de Geografía \\ Universidad Autónoma de Madrid
}

\section{RESUMEN}

Las sucesivas epidemias de cólera causaron millones de muertos en Europa en el siglo XIX, de los que unos 800.000 fallecieron en España. Se analiza aquí minuciosamente un caso concreto: el cólera en 1885 en la localidad cacereña de Garganta de Béjar, sus causas, desarrollo, precauciones, mortalidad, medicaciones, etc., bastante representativas de la situación sanitaria española y europea de entonces.

Palabras-clave: epidemia, cólera, Garganta de Béjar, 1885, geografía médica.

\section{ABSTRACT}

\section{The cholera epidemic in Garganta de Bejar (1885)}

The successive cholera epidemics caused millions of deaths throughout Europe in the $19^{\text {th }}$ century, 800.000 of these deaths occurred in Spain. One case in particular, the cholera epidemic of 1885 in the Caceres community of Garganta de Béjar, is analysed here in detail: the causes, development, precautions, mortality, medicines, etc., all of which are highly representative of the public health situation in Spain and Europe at the time.

Key words: epidemic, cholera, Garganta de Béjar, 1885, medical geography.

\section{INTRODUCCIÓN}

Las diversas "enfermedades infecto-contagiosas tuvieron un papel preponderante en la morbilidad y en la mortalidad ordinaria por lo menos hasta los últimos decenios del siglo XIX en toda Europa" (Pérez Moreda, 1980: 66-67). En España, a comienzos del siglo XX, aún suponían casi la cuarta parte de las defunciones (Revenga, 1904: 85), porcentaje elevado pero muy inferior al 52\% de los fallecimientos que seguían causando a nivel mundial (Rodríguez Cabezas y Rodríguez Idígoras, 1996: 108) y al 90\% de las muertes que habían generado en España hasta principios del siglo XIX, porcentaje que incitó a Vicente Pérez Moreda a calificar como "grandes asesinas del pasado" a enfermedades como la peste, el tifus, la viruela, el cólera, la fiebre amarilla y la fiebre recurrente (Pérez Moreda, 1980: 64-77).

Entre estas enfermedades se encuentra el cólera, transmisible por alimentos y agua (Olivera, 1993: 22), enfermedad que llegó a Europa entre 1817 y 1823 procedente de la India, convirtiéndose periódicamente en un azote para su población en el siglo XIX, ya que sus "víctimas en el continente europeo deben contarse por millones" (Fernández García, 1982: 7), generando varias oleadas de elevada mortalidad entre 1826 y 1911. Sheldon Watts señala que "el cólera surgió en forma epidémica en la India en 1817, y después de un falso arranque llegó a Gran Bretaña en 1831" (Watts, 2000: 229). Mientras las cinco epidemias de cólera provocaban en Gran Bretaña unas 130.000 muertes durante el siglo XIX, en India fallecieron, entre 1800 y 1925, entre 25,75 y 30,75 millones de personas, alcanzando su máxima mortalidad en 1900 con más de 800.000 muertos (Watts, 2000: 229 y 423).

Contacto: francisco.feo@uam.es 
A lo largo del siglo XIX invade, en sucesivas oleadas, todos los continentes: en 1817-1823 llega a Europa oriental y África, entre 1826 y 1836 a toda Europa, parte de África y América del Norte, en 1840-1855 a toda Asia, Europa, mitad norte de África y América del Norte y del Sur, en 1863-1869 a todo el mundo salvo Australia, en 1881-1885 a Asia, norte de África y Europa, y en 1892-1893 a estas mismas zonas y toda América (Cliff et al., 1981: 5; Cliff y Haggett, 1988: 3-11; y Haggett, 2000: 70). Solamente Australia quedó al margen del cólera a lo largo del siglo XIX.

Ya en 1885, José Montero señalaba que "el siglo XIX (...) ha hecho experimentar a Europa otra epidemia violenta y rápida, el cólera morbo asiático, que por desgracia sume actualmente en luto y desventuras sin cuento a casi toda España y comienza a propagarse por Francia e Inglaterra" (Montero Vidal, 1885 :37). En sólo los primeros 21 días de agosto de 1885 causó 636 defunciones en Marsella (Montero Vidal, 1885: 37). El cólera realizó invasiones continuas desde 1817 en países asiáticos, europeos, americanos, etc., penetrando en España por Vigo, Valencia, Barcelona y otros puertos costeros (Montero Vidal, 1885: 39-41).

Según Rodríguez Flores y Antona Rodríguez, "las incidencias cíclicas coléricas en el siglo XIX, en los años 1833, 1834, 1853, 1860 y 1885 provocaron niveles de mortalidad, en algunos casos, catastróficos, que significaron en la práctica, altísimos índices de mortalidad específica" (Rodríguez Flores y Antona Rodríguez, 1999: 18).

La epidemia de cólera de 1885 fue "menos intensa y con menos profusión por las zonas del interior que en años anteriores, presentó caracteres similares por mortalidad específica (7\%o), siendo el indicador a escala nacional de 37,9\%o)" (Rodríguez Flores y Antona Rodríguez, 1999: 20). Para estos mismos autores, "el cólera morbo no es una enfermedad moderna, sino muy antigua, pero pasó inadvertida hasta 1817, en que invadió Europa desde su cuna en la India, a orillas del Ganges (...). Era, desde el siglo XIX, una enfermedad endémica en la India y epidémica en el resto del mundo" (Rodríguez Flores y Antona Rodríguez, 1999: 125). Según estos mismos autores, "en 1854, el médico inglés John Snow demostró que el cólera se difundía mediante el suministro de agua" (Rodríguez Flores y Antona Rodríguez, 1999: 128) y, en 1883, Koch descubrió que el agente productor del cólera es el "vibrio comma arciforme" y que se transmitía principalmente por aguas contaminadas y por el contacto directo con los enfermos.

Su agente patógeno, el <bacilus vírgula > fue descubierto en 1883 por Robert Koch, científico prusiano (1843-1910), cuando residía en Alejandría y confirmó su hallazgo en 1884 en Calcuta. Aunque dos años antes había descubierto también el agente causal de la tuberculosis, sus teorías tardaron varias décadas en ser aceptadas y cuando lo fueron, permitieron a los médicos controlar la mayor parte de las epidemias (Rodríguez Cabezas y Rodríguez Idígoras, 1996: 95; Watts, 2000:15).

José María Moro ha sintetizado muy adecuadamente la situación de los conocimientos médicos sobre el cólera en la segunda mitad del XIX: "Hasta que Robert Koch descubrió en 1883 la bacteria causante del cólera (el vibrión colérico), las conjeturas acerca de las causas de la epidemia carecían de base sólida. Los médicos de la época, inicialmente apegados a la teoría miasmática, atribuían principalmente a las sustancias deletéreas emanadas de las materias orgánicas en putrefacción y transmitidos por el aire el origen de la epidemia y el medio de contagio; ello no excluía la convicción de que el contacto con los propios enfermos constituía un poderoso medio de propagación de la enfermedad. Pero estaban persuadidos también de que afectaba preferentemente a personas menesterosas, desnutridas y carentes de condiciones higiénicas, lo que se ponía claramente de manifiesto a la vista de la extracción social de la mayoría de los atacados por el mal. Efectivamente, no estaban descaminados, pues entre las causas de la invasión y extensión de la epidemia colérica dos factores sobresalen sobre cualesquiera otros: la insuficiente alimentación y la ausencia de higiene, tanto pública como privada" (Moro, 2003: 65).

Jaime Ferrán puso en práctica la vacuna anticolérica en 1884, si bien no sería reconocida oficialmente por medios internacionales hasta la reunión del Comité International d'Hygiène Publique celebrada en junio de 1919 en París (Pérez Moreda, 1980:76-77). En España, las aportaciones del doctor Ferrán fueron ampliamente criticadas por científicos y políticos, como analizó minuciosamente Juan José Fernández Sanz en su tesis doctoral (Fernández Sanz, 1989). 
En cualquier caso, a fines del siglo XIX parecía haber desaparecido en Europa y América y desde 1950 sólo se mantenía en la India y países próximos como Bangladesh, lo que no ha impedido epidemias de significativa importancia en la segunda mitad del siglo XX. Peter Haggett ha analizado su expansión geográfica entre 1961 y 1971: brota en el primer año en las islas Célebes, en 1962 se extiende al norte de Australia, en 1963 al sur de China e Indonesia, en 1964 a India, en 1965 a Irán, en 1970 a Oriente Medio, noreste de África y sur de Rusia, en 1971 a buena parte de África y algunas zonas del sur de España y Portugal (Haggett, 2000: 3).

La Organización Mundial de la Salud (OMS) registró en 1991 más de medio millón de casos de cólera, de ellos el 70\% en trece países de América Latina, alcanzando los 300.000 en Perú y cantidades menores en África (135.000 enfermos), Asia (12.568), Europa (311), Estados Unidos (24) y Canadá (2) (Olivera, 1993: 24-26). Entre 1991 y 1995, afectó a más de un millón de personas en América con 11.000 muertos, situación motivada por la contaminación de aguas de consumo y el uso de aguas residuales urbanas para regar cultivos, circunstancias que provocan, asimismo, la difusión de otras epidemias como tifus, disentería, malaria, etc. (Instituto de Recursos Mundiales, 2000: 24 y 51).

\section{CÓLERA EN ESPAÑA}

El cólera es una "enfermedad infecciosa exclusiva del ser humano. De origen indostánico, llegó a la Península Ibérica en 1835 a través del puerto de Vigo, desde donde se extendió por Galicia, Andalucía y Cataluña" (Anónimo, 1992: 2802). Según este anónimo autor, fue extendida por las guerras carlistas y "la peor epidemia de cólera en España tuvo lugar a finales de la década de 1850" y hubo otras en 1865 (Barcelona, Murcia, Sevilla y Madrid), en 1884 (costa mediterránea y espacios interiores), en 1890 (en el interior), 1971 (más de mil personas en toda España) y diversos casos aislados en periodos estivales (Anónimo, 1992: 2802).

José Montero señala que "en España, la primera invasión comenzó en Huelva el 9 de agosto de 1833 y concluyó en Ceclavín (Cáceres) el 31 de enero de 1835" (Montero Vidal, 1885: 44). El cólera supuso en España unos 300.000 fallecimientos en 1833-34, 236.000 en 1853-55, 120.000 en 1865 y 120.245 en 1885 (Fernández Sanz, 1990: 273).

Según José Montero, en 1885, "conocemos sobre 500 fórmulas, tratamientos y métodos preservativos y curativos del cólera, todos eficacísimos, según aseguran sus autores, muchos de los cuales gozan de gran reputación científica y profesional" (Montero Vidal, 1885: 74) aunque se inclina por precauciones generales de higiene, buen régimen alimenticio, vida moderada, rápido remedio al menor síntoma prodómico, desinfección de las habitaciones de enfermos, etc.

Entre marzo y agosto de 1885 se vieron afectadas en España 202.158 personas de las que 75.731 fallecieron por la epidemia de cólera, siendo Valencia la más afectada con 30.562 enfermos y 13.715 fallecidos (Montero Vidal, 1885:84-85). Según Rodríguez Flores y Antona Rodríguez, "durante el mes de julio de 1885 también Madrid, Zaragoza, Segovia, Ayamonte y La Granja padecen la epidemia, coincidiendo con las vacunaciones tan polémicas del Dr. Ferrán" (Rodríguez Flores y Antona Rodríguez, 1999: 154).

En Extremadura, hubo epidemias de cólera en 1833, 1853-1856, 1865, 1885 y 1890 (Merinero Martín, 1990: 168). Entre 1853 y 1856, se vieron afectadas unas 34.000 personas en Extremadura, de los que fallecieron 9.426 (Merinero Martín, 1990: 168). En 1865, los muertos ascendieron a 329 en Cáceres (Merinero Martín, 1990: 168). En 1885, en Badajoz, los afectados fueron 827 y los muertos 371 (Montero Vidal, 1885: 82-83). En la provincia de Cáceres la padecieron 72 (54 en Garganta de Béjar y 18 en El Gordo) y fallecieron 29 (22 en Garganta de Béjar y 7 en El Gordo) (Montero Vidal, 1885: 84-85). En 1885, en la provincia de Badajoz hubo 558 muertos (mayoritariamente del partido judicial de Don Benito) y en la de Cáceres fallecieron 57 (Merinero Martín, 1990: 168). María Jesús Merinero define el cólera como "enfermedad infecto-contagiosa, padecimiento epidémico más característico del siglo XIX en España. A lo largo de esta centuria, Extremadura conoció varias invasiones de la enfermedad. Su rasgo más notable era 
la rapidez e intensidad de su actuación. Se presentaba, y en el curso de unas semanas originaba una gran mortandad, de tipo catastrófico. El desconocimiento, en las primeras invasiones, de su mecanismo de actuación, y la facilidad del contagio, determinaban situaciones de histerismo colectivo, que contribuían a agravar los efectos del mal" (Merinero Martín, 1990:168).

En agosto de 1885 se padecía en Madrid, Cartagena, Albacete, Granada, Sevilla y Málaga y en otros países como Inglaterra, Francia y Portugal (Rodríguez Flores y Antona Rodríguez, 1999: 211). En la misma fecha el cólera se padece en Cáceres, habiendo en la capital acordonamientos y escasas condiciones sanitarias, finalizando con el mes en Don Benito y Villanueva (Badajoz) y en El Gordo, El Escurial y Garganta de Béjar (Cáceres) (Rodríguez Flores y Antona Rodríguez, 1999: 155 y 205). Extremadura sufre la epidemia en sus dos provincias, pero no en las capitales. La incidencia fue mayor en la de Badajoz que en la de Cáceres y, las consecuencias humanas y sociales fueron menores que en años anteriores" (Rodríguez Flores y Antona Rodríguez, 1999: 246). Estos mismos autores señalan que "1885 significó la última aparición del cólera. Ya Koch había descubierto el vibrión colérico" (Rodríguez Flores y Antona Rodríguez, 1999: 243) y "acordonamiento y vacuna fueron a lo largo de 1885 los dos aspectos más contestados, ambos fueron especialmente apreciados y también denostados" (Rodríguez Flores y Antona Rodríguez, 1999: 240). La Real Orden de 12 de junio de 1885 trató de combatir el cólera morbo con mayor higiene pública, medidas de aislamiento, lazaretos, desinfecciones, cuidados alimentarios y del agua, etc. (Rodríguez Flores y Antona Rodríguez, 1999).

\section{GARGANTA DE BÉJAR: UBICACIÓN Y MEDIO NATURAL}

Algo más de medio siglo antes de la epidemia, Pascual Madoz señala que Garganta de Béjar pertenecía a la provincia de Cáceres, partido judicial de Granadilla y diócesis de Plasencia y se ubicaba en una escarpada pendiente SE de las sierras que separan las provincias de Cáceres y Salamanca. Tenía un clima frío y vientos del Sur y Este. Limitaba al Noroeste con Baños (Salamanca), al Este con Béjar y Candelario (Salamanca) y al Sur con Hervás (Cáceres). Pascual Madoz constata que "este pueblo es uno de los agregados a la provincia de Cáceres en la última división territorial por exigirlo así su posición inclinada a las vertientes de Extremadura: se le llama alguna vez Garganta de Béjar" (Madoz, 1847, t. 8: 313). En 1991, Dionisia Gómez señala que tiene una extensión de 23,4 kilómetros cuadrados y que "casi todo el término municipal se halla por encima de los $1.000 \mathrm{~m}$., en las vertientes meridionales de la Sierra de Candelario, formando límite con la provincia de Salamanca" (Gómez Amelia, 1991: 130).

La fuente básica aquí utilizada es la escrita por Francisco Palacios Calderón, finalizada el 28 de octubre de 1885, fecha en la que envía su manuscrito "El cólera en Garganta de Béjar en el año de 1885" de 64 cuartillas a mano a la Real Academia de Medicina de Madrid para optar a uno de los premios que anualmente convocaba dicha Real Academia, organismo que conserva dicho manuscrito (signatura: $<1-3^{a}$ Pasillo 13-1). Como todas estas aportaciones se centra básicamente en el análisis del estado sanitario de la población (en forma de geografías médicas o de epidemias) de la zona estudiada, situación lógica al realizarlos médicos para presentarlos a los concursos anteriormente citados. Previamente, se realiza una descripción sobre el medio natural, población, actividades agrarias, etc., de los vecinos de dichos términos municipales, como realiza Francisco Palacios Calderón sobre Garganta de Béjar en el manuscrito que sirve de base a este estudio.

El doctor Palacios empieza ubicando Garganta de Béjar en una de las colinas que componen la cordillera Sierra de Béjar, derivación de las de Gredos. Está a $15 \mathrm{~km}$ de Béjar, a 9 de Candelario, a 10 de Hervás, a 4 de Baños de Montemayor y de Puerto de Béjar cuyos pueblos la circundan y todos se hallan a bastante menos altura que Garganta de Béjar que está a 1.500 metros sobre el nivel del mar aproximadamente. Su terreno es de sedimento correspondiente al grupo de areniscas. Palacios Calderón analiza algunos aspectos climáticos: dentro de las habitaciones, la temperatura media en invierno (de noviembre a mayo) es de unos $3^{\circ} \mathrm{C}$ y fuera hay nieves perpetuas, pasando de $9^{\circ}$ a $27^{\circ} \mathrm{C}$ en verano. El agua es excelente por ser manantiales o fuentes que con profusión se distribuyen excesivamente frescas en el verano y a placer en invierno. Hay muchísima humedad en la localidad por la profusión de manantiales 
en su alrededor y por la ausencia de limpieza del pueblo en invierno por la costumbre de hacer estiércol en todas las calles y patios, por convivir en las casas con dos o tres cabras, otros tantos cerdos y un asno en los ocho meses que en la localidad es invierno.

\section{SITUACIÓN ECONÓMICA, SOCIAL Y CULTURAL}

En 1847, Pascual Madoz había señalado que Garganta tenía "245 casas de dos pisos en 11 calles, todas en cuesta, empedradas, sin alineación ni limpieza, pero bañadas por aguas corrientes o regaderas construidas de intento y 1 plaza llana e irregular en el centro de la población, en la cual se halla la casa de Ayuntamiento, cárcel y escuela en un mismo edificio con las correspondientes separaciones; la última está dotada con 1.500 reales de los fondos públicos, y asisten 50 niños" (Madoz, 1847: 313). El mismo Madoz constata que "se surte de aguas potables en una buena fuente que hay en la plaza, con 2 caños, 1 pilón circular y buena cañería, de cuyo sobrante se alimentan las regaderas de las calles" (Madoz, 1847: 313).

Los 190 vecinos sumaban 1040 habitantes que producían vino, castañas, aceite, lino, legumbres, frutas, seda y escasos cereales por la poca tierra de labor. Se mantenía ganado cabrío, vacuno, lanar, de cerda, colmenas y se criaba mucha caza mayor y menor. Según Madoz, "los molinos harineros, telares de lienzo, elaboración de la exquisita manteca de vacas, venta y exportación de seda y frutas, entre las cuales es notable la manzana, e importación de granos" (Madoz, 1847: 313). El propio Madoz constató que había dos molinos harineros, telares de lienzo, una elaboración exquisita de manteca de vacas, vendía fruta (sobre todo, manzana) y seda e importaba granos. Pascual Madoz también apuntaba que "los caminos, aunque de sierra, están regularmente cuidados" y la escuela estaba dotada con 1.500 reales de fondos públicos y asistían 50 niños a ella (Madoz, 1847: 313). En la segunda mitad del siglo XIX se mantuvo estable la población: 1033 habitantes en 1900, incrementándose posteriormente hasta llegar a 1492 en 1950 y descendiendo a 738 en 1986, año en el que el 78,2\% de la población activa era agraria (Gómez Amelia, 1991: 130).

Según el doctor Palacios, en 1885, las principales producciones agrarias eran castañas, patatas, algo de centeno y muy pocas uvas. Dos tercios de sus habitantes trabajaban como jornaleros en los pueblos próximos y un tercio se dedicaban a la cría de cabras, algo de ganado porcino y muy pocos al vacuno. Casi un siglo después, "la economía agraria de la Tierra de Cáceres sigue descansando sobre los cultivos de cereales, la utilización ganadera de los pastos y la explotación del monte. Esta trilogía depende fundamentalmente de las condiciones naturales, y sobre todo del clima, cuya influencia es casi decisiva en una zona como ésta donde imperan técnicas agrarias tradicionales, a veces muy atrasadas" (Zulueta Artaloytia, 1977: 35).

Los servicios dejaban mucho que desear por el relieve accidentado y el aislamiento de la localidad, especialmente en invierno. Los medios de transporte y comunicación y acarreo eran en caballerías o andando. La higiene de los moradores era imposible en invierno y regular en los cuatro meses de verano. Para que fuera buena, el médico señala que sería necesario empezar por derribar las casas existentes. También critica la escasa educación porque el profesor abandona la escuela local porque los padres no mandan a sus hijos a ella.

El cementerio ha servido pero está a 15 metros de distancia de los moradores, con la particularidad de estar cerrado en su techo, ser muy pequeño, hallándose su suelo saturado de moléculas orgánicas en descomposición. Al cambiar en 1885 el Ayuntamiento, se consiguió lo que no se pudo con otros anteriores: empezando a construirse un nuevo cementerio a 300 metros de distancia, muy capaz y sin que pueda pervertir en lo más mínimo al pueblo. Se empezó con jornadas vecinales y algún jornal, estando parado por falta de recursos y, sin embargo, cuando el cólera se habilitó estrenándole con las dos hijas del médico.

El doctor Palacios no duda en calificar de momento crítico para el médico el serlo (más si fallecen dos hijas suyas) cuando empieza a extenderse una epidemia en su lugar de residencia y tiene que declararla a la autoridad cuando aún no se tiene muy claro que epidemia es y qué medidas tomar: alimentos a consumir y medicamentos. El problema se palió gracias a las 500 pesetas que enviaron la Diputación 
Provincial y el Gobernador para alimentar a los pobres, enfermos o sanos. Asimismo, el doctor Palacios Calderón hace hincapié en que la educación casi inexistente debería evitarse para que se propaguen las enfermedades, se diferencien algunas parecidas pero con soluciones distintas, etc.

\section{EPIDEMIA DE CÓLERA}

El 29 de julio de 1885, el doctor Palacios notificó al Alcalde que era necesario reunir la Junta de Sanidad y así se hizo, exponiendo el doctor Palacios los riesgos de las epidemias de cólera. Inmediatamente empezaron a tomarse precauciones higiénicas a pesar de contar con la opinión contraria del cura párroco que no notificó el peligro al Obispo cuando este podía socorrer a los pobres como otros prelados hicieron en sus respectivas diócesis en la epidemia de cólera de 1855. El doctor Palacios achaca esta forma de actuar del párroco a los 73 años del mismo, de los que los 33 últimos lo había sido en la localidad cuyos vecinos le creían más que al propio médico y que a todos los médicos juntos.

La epidemia afectó a 2 y 15 personas el 14 y 15 de agosto respectivamente que trató de combatir como médico y tratando de tranquilizar a enfermos y familiares de estos y del propio médico aunque el 17 de agosto se pidió por el sacerdote en la plaza del pueblo que se fuera el doctor Palacios a aplicar sus conocimientos sanitarios a Calcuta. Como consecuencia, la mayoría de los atacados por el cólera no llamaban al médico ni tomaban medicamentos ni aplicaban las propuestas higiénicas, lo que es duramente criticado por el médico de Garganta de Béjar a quien apoyó el maestro de instrucción primaria don Antonio García Merino, "un soldado voluntario siempre dispuesto al sacrificio y a secundar todo acto encaminado al bien de sus semejantes". También colaboró don Agustín Sánchez Hernández, secretario que animó y ayudó a enfermos y familiares a los que se ordenó aislamiento para tratar de frenar la expansión del cólera, aunque el doctor Palacios consideraba ineficaz el aislamiento de enfermos porque no se trasladan ni distribuyen medicamentos necesarios, lo que impide combatir dichas epidemias. El problema se acentúa en las clases proletarias que carecen de medios indispensables como alimentación sana y reparadora, habitaciones suficientemente amplias y en buenas condiciones higiénicas, fumigación y desinfección de las mismas, etc.

La invasión desde el 17 de agosto de la epidemia se extendió rápidamente y el 19 atacó al sacerdote al que visitó ese día el médico por hacer el maestro de intermediario entre ambos y curarse inmediatamente. El cura y otros afectados pasaron de considerar venenosos los medicamentos a tomarlos todos para tranquilidad del doctor Francisco Palacios. El apogeo de la epidemia llegó el 22 de agosto, día en el que el sacerdote Víctor Bruno Barrientos, sacerdote de Béjar, se situó en la cabecera de los enfermos sin arredrarse hasta que espiraron algunos matrimonios. Muchos que no avisaron al médico morían entre 24 y 36 horas desde que se vieron afectados y algunos incluso a las 8, 10 y 12 horas de contagiarse. Desde el 22 al 28 de agosto la epidemia fue decreciendo. El 30 reapareció con cinco casos leves pero que atemorizaron a los vecinos, mientras la llegada de don Santos Bredo, delegado del Gobernador, tranquilizó al médico al aportar 500 pesetas para socorrer a los indigentes aunque se vio afectado el doctor Palacios con la muerte de sus dos hijas, una de noche y la otra al día siguiente. El 5 de septiembre, el doctor Palacios descubre que él mismo padecía diarrea que se cura en los días siguientes con cantidades de nieve aportada por los vecinos, sustituyéndole el doctor Santos Oviedo en dichos días.

La epidemia de cólera duró del 28 de julio al 11 de septiembre, en total 46 días, muy por debajo de los 76 días que duró en Lillo (Toledo) y algo menos de los cincuenta que solía durar (Feo Parrondo, 2009: 179). Su llegada se atribuyó a un pastor que llegó a vender leche y queso al pueblo. Garganta de Béjar consumía poco alcohol, se alimentaba mal por ser carísima y de mala calidad: patatas, castañas y algo de pan de centeno y solamente carne en días festivos.

Se atribuye parte de la epidemia a su procedencia de Salamanca, La Calzada, Peñaranda y del río Tormes que estaba infectado y plagado de microbios colerígenos. Asimismo, se achaca a algún pastor procedente de la Sierra de Gredos y a que los vecinos habían comido carne de un toro en malas condiciones procedente del pueblo de Hervás. La difusión se acentúa por ser habitaciones pequeñas, contiguas, húmedas... con el consiguiente hacinamiento en casa, calles estrechas, etc. 
El doctor de Garganta de Béjar cita los síntomas: se suele iniciar mayoritariamente con diarrea premonitoria, vértigos, escalofríos y gran debilidad. No obstante, se suele notar inicialmente con dolores gástricos de pronto, a media noche, con vómitos, deyecciones, diarreas profusas, nauseas, sed abrasadora, supresión de orina, lengua húmeda y blanquecina, debilidad, hipotermia periférica, calambres, etc. Tras estos síntomas aparece el periodo álgido con menor temperatura en piel, lengua y aire expirado, extinción de la voz, etc., iniciando la agonía. Suelen durar entre 16 y 20 horas para llegar a la terminación fatal, cifra que, incluso hoy en día, sería difícil de combatir en núcleos urbanos y, más en 1885, en un núcleo rural de zona montañosa como Garganta de Béjar. De los 239 afectados se curaron 93 con salierato de bismuto y el laudeno, otros 95 se vieron poco afectados y 51 fallecieron por las pésimas condiciones higiénicas y/o por no avisar al médico.

Los casos observados no reunían la sintomatología completa del cólera morbo asiático, predominando la diarrea, vómitos y calambres. La razón apuntada es que se extendía lentamente salvo cuando bajaba la temperatura y porque disminuía sus invasiones y fallecimientos al poco tiempo de invadir localidades. A finales del verano y principios del otoño de 1884 había afectado a las provincias del Levante español con pocos fallecimientos pero extendiéndose rápidamente y siendo frecuente que vuelvan a reaparecer el verano siguiente después de que el frío invernal aletargara los gérmenes del cólera. Este temor también lo apunta como posible para Garganta de Béjar por lo que pide a las autoridades tomar medidas preventivas.

Para el médico de Garganta de Béjar, el primer problema es detectar qué enfermedad llega a la zona en que ejerce su profesión. Para el cólera, considera un síntoma fiable el que "un individuo tendido en la cama u otro sitio, con los ojos extraordinariamente abiertos, muy hundidos y rodeados de una extensa aureola violácea o negruzca, con la mirada extraviada, nariz afiladísima, labios entreabiertos, la voz casi afónica, la lengua ancha, húmeda y blanquecina y en todas sus facciones retratándose el colmo de la ansiedad, el summun del sufrimiento, estremeciéndose y retorciéndose todo su cuerpo, con ese frío periférico tan característico, sin pasar a mas averiguaciones con independencia y con valor podremos decir que es el cólera" (pp. 43). El proceso se acentuaba si la familia señalaba que el enfermo tenía vómitos, diarrea, calambres, supresión de orina, etc.

Para el médico de Garganta de Béjar, "la verdadera medicina es la higiene, prevenir los males antes que curarlos" y "el abatimiento producido por el miedo es el primer y más eficaz factor del cólera" (pp. 44). Hasta que no se compruebe realmente que es el cólera, no se debe vacunar a los enfermos y el anónimo médico duda de dicha vacuna al inicio de las epidemias, señalando que "el cólera es menos terrible que otras enfermedades cuando se desarrolla epidémicamente como la viruela y el tifus, siempre que se llame a tiempo y viviendo bajo ciertas condiciones higiénicas y de ánimo, demandando pronto el auxilio de la Medicina, se curan por lo menos las tres cuartas partes de los atacados" (pp. 45).

Asimismo, señala que "cuando el cólera se ha desarrollado en una localidad, el ánimo debe estar tranquilo y sereno; la pusilaminidad es mala compañera, casi siempre es la causa predisponente y ocasional de esta enfermedad" (pp. 45-46). También señala que "la alimentación debe ser sana, sencilla y sobria, sin condimentos excitantes, la carne asada debe ser preferida a cualquier otro guiso; pueden usarse las frutas ácidas, bien maduras y en poca proporción y después de las comidas, quesos o pastas o las dos cosas" (pp. 46). Igualmente apunta que "debe prescribirse el uso moderado de las bebidas alcohólicas y del café y té. El vino a las comidas debe ser del de fiasto o tinto seco y mejor (donde esto puede hacerse) aún si le mezclamos con agua de sel" (pp. 46). No duda en afirmar que "no debe hacerse uso de sustancias ni remedios preservativos al interior, pues además de que de nada sirven, son más o menos alterantes y suelen perjudicar" (pp. 46).

"El alcanfor es mi medio preservativo de muy buenos efectos: destruye los agentes morbosos vegetales y animales microscópicos y es el mejor factor para prevenir y combatir los accidentes nerviosos" (pp. 46 - 47). El médico aconseja que se aspire a menudo y llevarlo en el bolsillo interior de la ropa. Asimismo, propone que se añada al agua de lavarse una cucharada de vinagre o de ácido acítico. Las medidas higiénicas que sugiere, incluyen también ventilar bien las habitaciones y desinfectarlas con gas hiponítrico, aseo del cuerpo y de la cama y dos veces diarias de las letrinas, etc. 
En los medios farmacológicos propone aguardiente y en cuanto se presente la diarrea el médico propone tomar 10 ó 12 gotas de lacídano en un terrón de azúcar o en dos dedos de agua e, inmediatamente meterse en la cama, abrigarse, tomar una taza de manzanilla, menta o te bien caliente, a la que se añade una copita de infusión o maceración del aguardiente. Si el enfermo suda es que está salvado pero debe permanecer en la cama dos o tres días con caldo, arroz y alimentos tenues y sencillos.

Si sigue la epidemia y toma alarmantes proporciones convirtiéndose en diarrea con vómitos y sed intensa, se le administra una píldora cada cuatro horas, algo de nieve que calma la sed y de aguardiente que apacigua los calambres. Si no se curasen se aplicarían inyecciones hipodérmicas estimulantes para atacar los síntomas más elevados. El agua debe beberse fría, natural, en pequeñas cantidades o, incluso, sustituirse por nieve en pequeñas cantidades y de pequeño tamaño como había comprobado personalmente en su estómago el anónimo médico de Garganta de Béjar, quien llegó a informar a su familia que no se moría.

Para el doctor Francisco Palacios, "el gobierno debe llevar a efecto la cremación de los cadáveres con energía y sin miramiento ni contemplación alguna, y está por encima de todas las leyes sociales y religiosas, y de todas las exigencias y prevenciones injustificadas" (pp. 53).

En Garganta de Béjar fueron invadidas 146 personas con el cólera: 57 mujeres, 42 hombres, 25 niñas y 22 niños (pp. 62). De ellos, fallecieron 51 personas: 18 mujeres, 15 hombres, 7 niñas y 11 niños (pp. 63).

Francisco Palacios y Calderón acompaña el trabajo sobre el cólera con una descripción interesante sobre la valoración que se hacía entonces de los médicos: "el médico que ejerce su sacerdocio, el que se emplea en devolver la salud a sus semejantes cumple un deber sagrado que se ha impuesto y desde el primer enfermo hasta el último que visita, se halla en lucha perpetua con la muerte o sea con el cambio de vida, pues en la naturaleza nada se pierde" (pp. 54). Tras enumerar algunos riesgos de contagio, señala que "en esta guerra continua con la enfermedad, el médico es siempre el héroe anónimo en las sociedades, y la recompensa y aprecio que recibe muchas veces, es bien mezquino y miserable, si no vence la enfermedad es despachado con desdén de los enfermos y familia de estos, pues siendo más los ignorantes que las gentes de entendimiento, no ven la impotencia acaso de la medicina, y se lo achacan a la falta de tacto y saber del médico" (pp. 56). Si el enfermo se cura se le achaca a un santo y el doctor Palacios apunta que se podrían haber reducido en 8 o 10 los muertos de la localidad si hubieran tomado los medicamentos sugeridos por el doctor que señala que están poco valorados por la sociedad española.

\section{BIBLIOGRAFÍA}

ANÓNIMO (1992): "Cólera" en Gran Enciclopedia de España, Navarra, Estella, t. 6, pp. 2802.

CLIFF, A.D. et al., (1981): Spatial diffusion: an historical geography of epidemics in an Island Community, Cambridge, University Press, 286 págs.

CLIFF, A.D. y HAGGETT, P. (1988): Atlas of disease distributions: analytic approaches to epidemiological data, Oxford, Blackwell Reference, 300 págs.

FEO PARRONDO, F. (1996): Geografías médicas de Tineo de 1886, 1907 y 1913, Oviedo, Principado de Asturias, 176 págs.

FEO PARRONDO, F. (2005): "La epidemia de cólera en San Fernando de Henares (1865)”, Nimbus, 15-16, pp. 57-72.

FEO PARRONDO, F. (2009): "La epidemia de cólera de 1885 en Lillo (Toledo)", Papeles de Geografía, 49-50, pp. 175-179.

FEO PARRONDO, F. (2011): "Geografía médica y epidemias en Villarta de los Montes (Badajoz) (1904-1912)", Nimbus, 27-28, pp. 5-24.

FEO PARRONDO, F. (2011): "Epidemias en la provincia de Segovia (1909-1923)", Boletín de la Real Sociedad Geográfica, 147, pp. 131-148. 
FERNÁNDEZ GARCÍA, A. (1982): El cólera de 1885 en Madrid, Madrid, Ayuntamiento, 48 págs. FERNÁNDEZ GARCÍA, A. (1985): Epidemias y sociedad en Madrid, Barcelona, Vicens Vives, 274 págs. FERNÁNDEZ SANZ, J. J. (1989): El cólera de 1885 en España, Madrid, Universidad Complutense, 738 págs. FERNÁNDEZ SANZ, J. J. (1990): 1885: El año de la vacunación Ferrán. Transfondo político, médico, sociodemográfico y económico de una epidemia, Madrid, Fundación Ramón Areces, 410 págs.

GÓMEZ AMELIA, D. (1991): "La Garganta", Gran Enciclopedia Extremeña, Mérida, Edex, t. 5, 130 pp.

GURRIA GASCÓN, J. L. et al., (1999): "La población extremeña en el tránsito del siglo XIX al XX", Revista de Estudios Extremeños, t. 1, pp. 265-314.

HAGGETT, P. (2000): The geographical structure of epidemics, Oxford, University Press, 150 págs.

LLORENTE DE LA FUENTE, A. (1993): La epidemia de cólera de 1885 en Valladolid y provincia, Valladolid, Universidad.

MADOZ, P. (1847): "Garganta” en Diccionario geográfico-estadístico-histórico de España y sus posesiones de Ultramar, Madrid, t. 8, 313 pp.

MERINERO MARTÍN, M. J. (1990): "Cólera”, Gran Enciclopedia Extremeña, Mérida, Edex, t. 10, 168 pp.

MONTERO VIDAL, J. (1885): El cólera en 1885, Madrid, Manuel Hernández, 144 págs.

MORO, J. M. (2003): Las epidemias de cólera en la Asturias del siglo XIX, Oviedo, Universidad, 306 págs.

OLIVERA, A. (1993): Geografía de la salud, Madrid, Síntesis, 160 págs.

PERAL PACHECO, D. (1994): Cólera y sanidad en las Reales Ordenes de 1833 a 1855, Mérida, Asamblea de Extremadura, 140 págs.

PÉREZ MOREDA, V. (1980): La crisis de la mortalidad en la España interior, siglos XVI-XX, Madrid, Siglo XXI, 526 págs.

REVENGA, R. (1904): La muerte en España. Estudio estadístico sobre la mortalidad, Madrid.

RODRÍGUEZ CABEZAS, A. y RODRÍGUEZ IDIGORAS, M.I. (1996): Historia ilustrada de la Medicina, Málaga, Algazara, 142 págs.

RODRÍGUEZ FLORES, M. P. y ANTONA RODRÍGUEZ, M. J. (1999): La percepción de la epidemia de cólera de 1885. Badajoz ante una crisis, Cáceres, Universidad de Extremadura, 254 págs.

RODRÍGUEZ OCAÑA, E. (1981): Enfermedad y sociedad en la Andalucía del siglo XIX: la epidemia de cólera de 1833-35, Granada, Universidad, 28 págs.

URTEAGA, L. (1980): "Miseria, miasmas y microbios. Las topografías médicas y el estudio del medio ambiente en el siglo XIX", Geocrítica, 29, 50 págs.

UTANDA MORENO, L. (2001): "La epidemia de cólera en Madrigal de las Altas Torres en 1885", Cuadernos Abulenses, 30, pp. 177-196.

WATTS, S. (2000): Epidemias y poder. Historia, enfermedad, imperialismo, Barcelona, Andrés Bello, 492 págs.

ZULUETA ARTAlOYTIA, J. A. de (1977): La Tierra de Cáceres. Estudio geográfico, Madrid, CSIC-UAM, 180 págs. 